\title{
Hong's grading for evaluating anterior chamber angle width
}

\author{
The Editors, Japanese Journal of Ophthalmology
}

Published online: 9 July 2013

(C) Japanese Ophthalmological Society 2013

\section{To the Editor}

I read with interest and concern the article by Kim and colleagues entitled, "Hong's grading for evaluating anterior chamber angle width" in the November/December 2012 issue of the Japanese Journal of Ophthalmology [1].

I was glad to read the comment that said, "Hong's grading cannot substitute for angle evaluation by gonioscopy;..." However, I was not so happy with the concluding portion of that sentence which was, "... however, in cases where a gonioscope or an experienced examiner are not available, Hong's grading can provide an alternative method." I don't believe that this article confirms that.

There are very real concerns that anterior segment optical coherence tomography (AS-OCT) will come to replace gonioscopy. It is not a substitute. It has many advantages, which are well discussed in Kim et al.'s study. In fact, comparing AS-OCT to gonioscopy is like comparing a black and white photograph of the Grand Canyon to walking through the canyon; the AS-OCT and the photograph provide information that is "standardized," but the big picture is completely missing.

I was also deeply concerned by the statistical evaluation of the data. It was marvelous that the authors provided real data points, in the scattergrams shown on page 555. Indeed, regression lines could be drawn through these. But, these regression lines are highly misleading. The major point to be drawn from the scattergrams on page 555 is that very few of the points fall on the regression line and that the correlations are shaky at best. This is even more striking in Figure 5. If the one data point showing an intraocular

The Editors, Japanese Journal of Ophthalmology $(\bowtie)$

Tokyo, Japan

e-mail: jjo@po.nichigan.or.jp pressure of $1 \mathrm{mmHg}$ in an angle which is graded as 60 using Hong's method is removed, what is immediately apparent is that there is no relationship between the width of the angle and intraocular pressure. Indeed that's what one would expect. Presumably an open angle is open, and whether it's narrow or wide would not have any particular effect on intraocular pressure. But that the authors' conclusion that angle width correlates with likelihood of pressure rise in a darkroom provocative test is unfortunate and misleading.

Sincerely,

George L. Spaeth, M.D.

Wills Eye Institute/Jefferson Medical College, Philadelphia, Pennsylvania, USA

\section{Author's Reply}

We appreciate the interest and concern that Dr. Spaeth has shown in this study.

We agree with Dr. Spaeth's comments that 'Anterior segment optical coherence tomography (AS-OCT) cannot be a substitution of gonioscopy.' Gonioscopy is the current gold standard for evaluation of the anterior chamber angle and we believe that AS-OCT cannot replace gonioscopy in this respect. However, AS-OCT has unique advantages over gonioscopy. It provides standardized and quantitative information on angle structure. Therefore, in this study, we compared Hong's angle width with the AS-OCT parameters, instead of with gonioscopic gradings. In addition, as we mentioned in the Discussion, we do not believe that Hong's grading can substitute for gonioscopic evaluation. Rather, we believe that similar to the van Herick method, 
Hong's method is useful as a screening tool to identify patients at risk of angle closure.

In the correlation analysis, it is not possible that the majority of the data points in this study fall on the regression line because Hong's method estimates the angle width by indirect calculation and overestimates the anterior chamber angle width in eyes with an open angle. Nevertheless, measurements of angle width performed using Hong's method showed a statistically significant linear correlation with values obtained using the AS-OCT. The dark room prone position test (DRPT) was performed in 26 subjects. Among these 26 subjects, only 4 had eyes with an open angle. If we remove those 4 eyes with an open angle from the correlation analysis including the outlier with Hong's angle width of 60 , there remains a highly significant linear correlation $(r=-0.659$, $P<0.01)$. Therefore, we think that Hong's angle width is significantly associated with IOP increases after DRPT in eyes with a narrow angle.
Seok Hwan Kim, MD

Department of Ophthalmology, Boramae Medical Center,

Seoul, Korea

Ki Ho Park, MD

Department of Ophthalmology,

Seoul National University College of Medicine,

Seoul, Korea

\section{Reference}

1. Kim SH, Kang JH, Park KH, Hong C. Hong's grading for evaluating anterior chamber angle width. Jpn J Ophthalmol. 2012;56:551-8. 